\title{
THE EFFECTS OF STRAIN ANNEALING ON GRAIN BOUNDARY DISTRIBUTION AND HARDENING IN SUPERPURE NICKEL
}

\author{
C. B. THOMSON and V. RANDLE \\ Department of Materials Engineering, University of Wales Swansea, \\ Singleton Park, Swansea SA2 8PP, UK
}

(Received 10 March 1996)

\begin{abstract}
Electron backscatter diffraction is applied to the study of texture and mesotexture in superpure nickel. Low level strain annealing is shown to influence the grain boundary population such that greater proportions of special boundaries exist. It is found that variations in the texture of a specimen are not reflected by characteristic changes in the grain boundary population, indicating that texture analysis cannot be applied to the prediction of special boundary densities. Mechanisms active during the evolution of special boundaries are discussed and compared to those involved under similar conditions in commercially pure nickel. It is shown that annealing twin formation need not be prevalent for a high level of special boundaries to form. Differences in the hardness of various boundary types are identified, such that low angle boundaries and $\Sigma 3$ boundaries close to exact misorientation can be categorized separately to other boundaries, in that they show minimal hardening.
\end{abstract}

KEY WORDS: Nickel, texture, mesotexture, special boundaries, EBSD.

\section{INTRODUCTION}

A high degree of association exists between the structure and many properties of grain boundaries. Since the grain boundary network in a polycrystal exists in a metastable state, it is possible to manipulate the boundary population in order to introduce higher proportions of boundaries with superior properties, i.e. 'special' boundaries. Strain annealing is one approach that has been applied to induce such an effect (e.g. Lim and Raj, 1984a; Palumbo and Aust, 1990; Crawford and Was, 1991).

The degree of plastic strain and annealing temperature are two factors that influence the final grain boundary distribution. If the annealing temperature is sufficiently high, grain boundary migration can be activated as a process by which the overall free energy is reduced. If, however, the temperature is insufficient to initiate grain growth, some of the dislocations introduced via the initial strain can be absorbed into boundaries and dissociate into extrinsic grain boundary dislocations (EGBDs). These provide a driving force for the grain or grain boundary to rotate to a more energetically stable configuration (Randle and Brown, 1989; Chiba et al., 1994). Such rotations are hampered by the presence of precipitates at the boundary, introducing a drag factor and hence increasing the energy required to activate this mechanism. It has been shown previously, by Thomson and Randle, 1996a, that for such a situation in commercially pure $(99.5 \%)$ nickel, an alternative grain boundary energy minimization process predominates, namely annealing twin formation. The primary purpose of the 
present work is to compare the energy minimization processes occurring in superpure nickel with results from the commercial grade material.

A second aim of the present work is to monitor a specific property which is grain boundary structure-dependent, and to relate this to the evolving grain boundary geometry distribution. The phenomenon of grain boundary hardening is such a property. It is considered to be affected by solute segregation (e.g. Watanabe, Kitamura and Karashima, 1980; Chou et al., 1983) in addition to grain boundary structure. Although initial impurity levels are at 10 parts per million atoms in the superpure nickel investigated here, the thermal treatments were conducted in air, which will introduce high levels of oxygen and other species to the grain boundary network. Segregation of these impurity atoms to the grain boundaries will exaggerate any boundary hardening due to structural effects alone. Measurements of microhardness variations a grain boundaries are reported and discussed here.

\section{EXPERIMENTAL}

The thermomechanical treatment regime chosen for this experimental programme on cold rolled superpure nickel was selected by the requirement to promote a small driving force for strain induced grain growth, and to avoid initiating recrystallization. $0.5 \mathrm{~mm}$ thick specimens of $99.999 \%$ purity nickel were compressively strained to $6 \%$. Two-stage thermal treatments were then applied in an air atmosphere. The first stage $\left(9\right.$ hours $\left./ 500^{\circ} \mathrm{C}\right)$ allowed oxygen to diffuse into the sample. After cooling to room temperature, the second stage was conducted at $850^{\circ} \mathrm{C}$, with different treatment times for each sample. This second stage temperature is just sufficient to initiate grain growth. The treatment details are given in Table 1.

The specimens were prepared, using standard metallographic techniques, for electron backscatter diffraction (EBSD) in a JEOL 6100 SEM. Highly polished final surfaces were produced using a $0.06 \mu \mathrm{m}$ colloidal silica suspension, followed by electrolytic etching in $70 \%$ phosphoric acid to reveal the grain boundaries. The EBSD results were processed to allow orientation and misorientation analysis to be conducted. Grain sizes were evaluated via linear intercept measurements from the SEM micrographs. Finally, with the predetermined grain boundary misorientations as prior knowledge, boundary hardening measurements were made using a pyramidal indenting LECO M-400-G1 hardness tester. A $10 \mathrm{~g}$ constant load, the minimum available, was applied for five seconds per measurement, and an average of four indents per grain/ grain boundary was calculated.

Table 1 The processing details for the four superpure nickel samples studied.

\begin{tabular}{lcccc}
\hline & Sample 1 & Sample 2 & Sample 3 & Sample 4 \\
\hline $\begin{array}{l}\text { Compressive } \\
\text { Strain (\%) }\end{array}$ & None & 6 & 6 & 6 \\
\hline $\begin{array}{l}\text { Thermal } \\
\text { Treatment }\end{array}$ & None & $\begin{array}{l}9 \mathrm{Hr} / 500^{\circ} \mathrm{C}+ \\
8 \mathrm{Hr} / 850^{\circ} \mathrm{C}+\end{array}$ & $\begin{array}{l}9 \mathrm{Hr} / 500^{\circ} \mathrm{C}+ \\
48 \mathrm{Hr} / 850^{\circ} \mathrm{C}\end{array}$ & $\begin{array}{l}9 \mathrm{Hr} / 500^{\circ} \mathrm{C}+ \\
168 \mathrm{Hr} / 850^{\circ} \mathrm{C}\end{array}$ \\
\hline
\end{tabular}




\section{RESULTS}

Figure 1 shows the $\{111\}$ pole figure for each sample. Sample 1 , as it is untreated after cold rolling, reveals a typical rolling texture. As the treatment time is increased, from sample 1 to 4 , the rolling texture is seen to diminish. For sample 1 the copper, $\mathrm{S}$ and brass textures, which are then main rolling components, are present in the proportions $6 \%, 16 \%$ and $22 \%$ respectively. Although the characteristic rolling texture diminishes with increased thermal treatment time, two of the main component textures actually increase in prominence. The $S$ texture increases to $44 \%$ and the copper texture to $23 \%$. Other prominent changes that occur with increased treatment times are in the cube texture, which drops from $11 \%$ in sample 1 to $4-5 \%$ for samples 2 to 4 , and the (211)[011] texture which falls from a similar level of $10 \%$ (sample 1) to about $1 \%$ (samples 2 to 4 ). The only other texture type that shows any sensitivity to increased thermal treatment is the (103)[010], which changes from 2-3\% for samples 1 to 3 , to $7 \%$ for sample 4 .

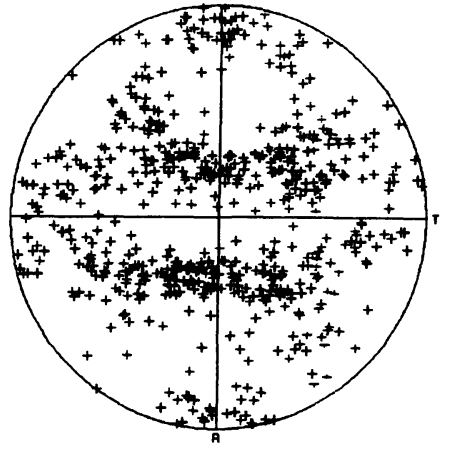

(a)

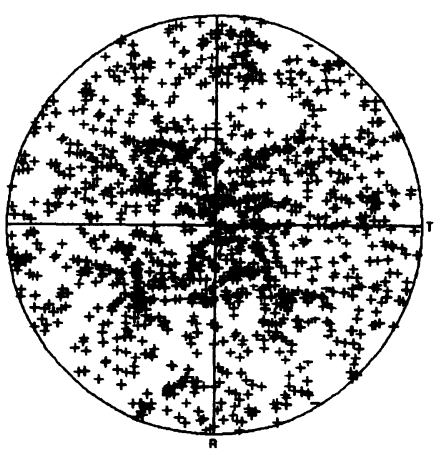

(c)

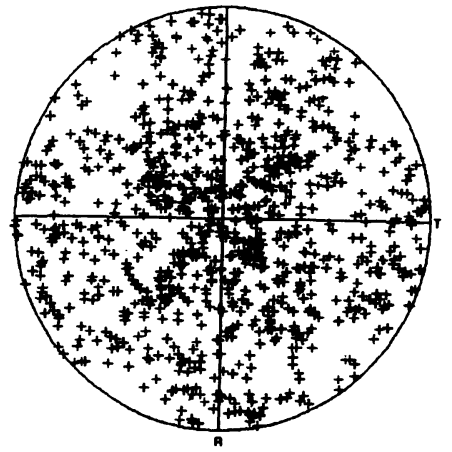

(b)

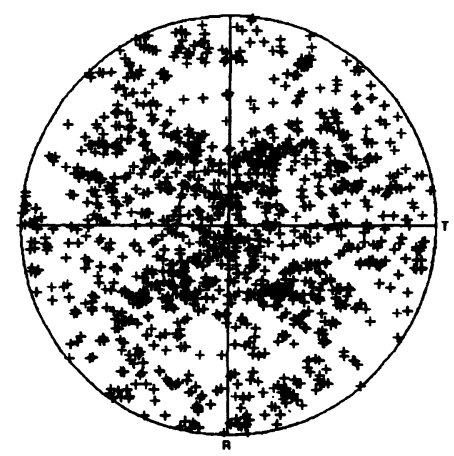

(d)

Figure $1\{111\}$ pole figures for the untreated specimen (a) and the three strain annealed samples in order of increasing treatment time at $850^{\circ} \mathrm{C}$, (b) to (d) respectively. 
Table 2 The proportion of low $\Sigma$ CSLs $(\Sigma \leq 35)$ present in the total grain boundary population. The mean grain size is also given for each of the four samples studied.

\begin{tabular}{|c|c|c|c|c|c|c|c|c|c|c|}
\hline $\begin{array}{l}\text { Sample } \\
\text { ID }\end{array}$ & $L A B$ & $\Sigma 3$ & $\Sigma 5$ & $\Sigma 7$ & 29 & $\Sigma 11$ & $\Sigma 13$ & $\Sigma 15$ & $\Sigma 17$ & $\Sigma 19$ \\
\hline $\begin{array}{l}1 \\
2 \\
3 \\
4\end{array}$ & $\begin{array}{l}6.32 \\
3.23 \\
2.56 \\
3.50\end{array}$ & $\begin{array}{r}7.69 \\
21.39 \\
28.10 \\
29.46\end{array}$ & $\begin{array}{l}0.27 \\
0.85 \\
1.92 \\
1.92\end{array}$ & $\begin{array}{l}2.47 \\
0.85 \\
1.60 \\
1.69\end{array}$ & $\begin{array}{l}1.37 \\
2.38 \\
4.81 \\
3.84\end{array}$ & $\begin{array}{l}1.65 \\
0.85 \\
0.64 \\
1.47\end{array}$ & $\begin{array}{l}0.82 \\
0.68 \\
0.85 \\
1.12\end{array}$ & $\begin{array}{l}0.27 \\
0.51 \\
0.96 \\
0.90\end{array}$ & $\begin{array}{l}1.10 \\
1.02 \\
0.75 \\
0.90\end{array}$ & $\begin{array}{l}0.27 \\
1.02 \\
0.11 \\
0.45\end{array}$ \\
\hline $\begin{array}{l}\text { Sample } \\
I D\end{array}$ & $\Sigma 21$ & $\Sigma 23$ & $\Sigma 25$ & $\Sigma 27$ & $\Sigma 29$ & $\Sigma 31$ & $\Sigma 33$ & $\Sigma 35$ & $\begin{array}{c}\text { Mean } \\
\text { Size }\end{array}$ & $\begin{array}{l}\text { Grain } \\
(\mu m)\end{array}$ \\
\hline $\begin{array}{l}1 \\
2 \\
3 \\
4\end{array}$ & $\begin{array}{l}0.27 \\
1.19 \\
1.28 \\
1.02\end{array}$ & $\begin{array}{l}0.27 \\
0.85 \\
0 \\
0.45\end{array}$ & $\begin{array}{l}2.75 \\
0.68 \\
0.96 \\
1.13\end{array}$ & $\begin{array}{l}0.82 \\
1.19 \\
1.18 \\
1.70\end{array}$ & $\begin{array}{l}0.27 \\
0.85 \\
0.53 \\
0.34\end{array}$ & $\begin{array}{l}0.27 \\
0.68 \\
0.53 \\
0.45\end{array}$ & $\begin{array}{l}0.82 \\
1.02 \\
0.42 \\
0.79\end{array}$ & $\begin{array}{l}0.82 \\
0.51 \\
0.42 \\
0.34\end{array}$ & $\begin{array}{r}29 \\
73 \\
92 \\
121\end{array}$ & \\
\hline
\end{tabular}

Approximately 800 grain boundary misorientations were evaluated per specimen. The only CSL types affected by increasing the processing are twin-related boundaries $(\Sigma 3, \Sigma 9, \Sigma 27)$, as can be seen in both Table 2 and Figure 2 . A slight drop in low angle boundary (LAB) numbers can also be observed. Extending the treatment time at $850^{\circ} \mathrm{C}$ increases the proportion of $\Sigma 3 \mathrm{~s}$ in the grain boundary network to an upper limit. Further analysis of the $\Sigma 3$ data reveals that not only does the total $\Sigma 3$ content increase with treatment time, but the proportion with low deviations from exact misorientation also increases significantly. These observations are presented in Figure 3 in terms of $v / v_{m}$, the deviation from exact misorientation, where $v$ is the measured deviation and $\mathrm{v}_{\mathrm{m}}$ is the maximum permitted deviation defined by the Brandon criterion (Brandon, 1966). This is a significant result because of the correlation between low deviation from exact misorientation and lower grain boundary free energy, and the associated special properties of such boundaries (Pumphrey, 1976). The energy of a coherent twin in nickel, which would be close to exact $\Sigma 3$ misorientation, is $0.027 \mathrm{Jm}^{-2}$ whereas that for an incoherent twin in nickel, which is further from the exact $\Sigma 3$ misorientation, is $0.606 \mathrm{Jm}^{-2}$. The average high angle grain boundary energy in nickel is $0.860 \mathrm{Jm}^{-2}$ (Murr, Smith and Gilmore, 1968).

The measured grain sizes are given in Table 2 and show that although grain boundary migration is active, it is not dominant as a method of grain boundary energy minimization. This is very likely to be a consequence of drag effects hampering grain boundary mobility, as a result of the presence of precipitates at the boundaries. The grain growth law equation can be written as:

$$
\log \left(D-D_{o}\right)=n \log (t)+k
$$

where $D_{0}$ is the initial mean grain size, $D$ is the mean annealed grain size, $n$ is the grain growth exponent, $t$ is the treatment time and $k$ is a constant for constant temperature. Figure 4 shows a plot of $\log \left(D-D_{o}\right)$ against $\log (t)$ for the data obtained here, the gradient of which gives the grain growth exponent. The ideal grain growth 


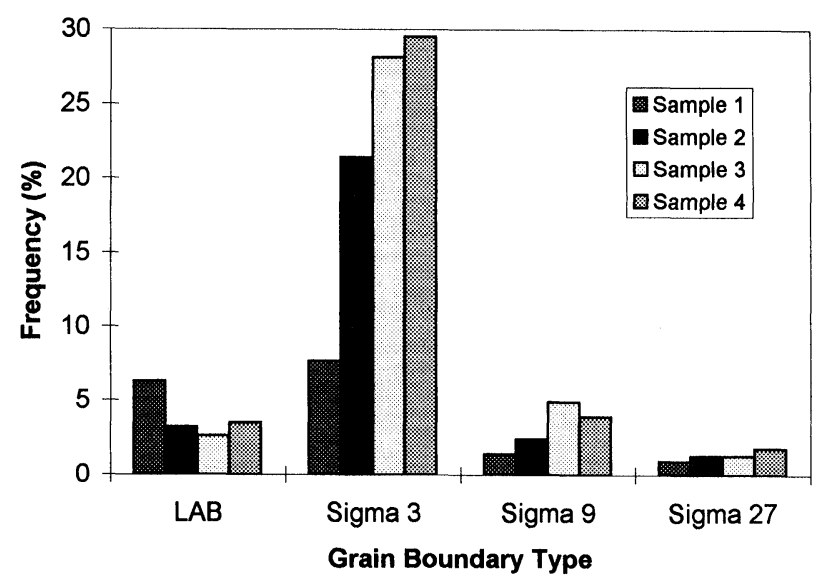

Figure 2 The proportion of twin-related CSLs in the grain boundary population.

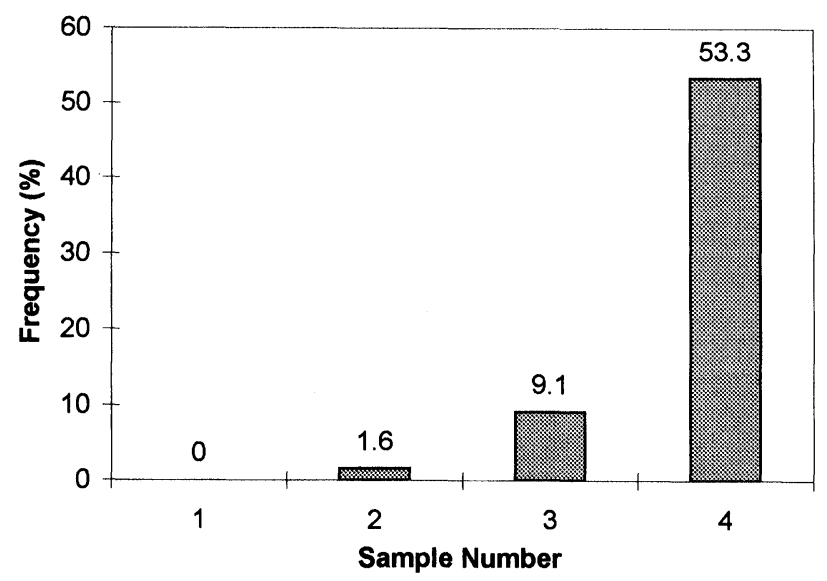

Figure 3 The proportion of low-deviation $\Sigma 3 \mathrm{~s}$ in the total $\Sigma 3$ population.

law presents grain size as proportional to the square root of time, i.e. $n=0.5$. From Figure 4 the grain growth exponent for this experiment is evaluated as 0.25 . This clearly indicates a strong reduction in grain boundary migration rates.

Figure 5 shows the results from the grain boundary hardening study of sample 4 . The data are presented in terms of the following categories of boundary: LAB; low deviation $\Sigma 3\left(\mathrm{v} / \mathrm{v}_{\mathrm{m}}<0.2\right)$, or LD $\Sigma 3$; high deviation $\Sigma 3\left(\mathrm{v} / \mathrm{v}_{\mathrm{m}}>0.2\right)$, or HD $\Sigma 3$; $5 \leq \Sigma \leq 35$; and random high angle boundary (RHAB). In each case seven boundaries were studied. The Vickers hardness values for the boundary and adjoined grains are given in each case. The results obtained for LABs show that the hardness at the boundary is typically no greater than in one or both of the adjacent grains. A similar result is obtained for LD $\Sigma 3 \mathrm{~s}$. However, for other CSL types, including HD $\Sigma 3 \mathrm{~s}$, and RHABs there appears to be measurable hardening at the grain boundary. 


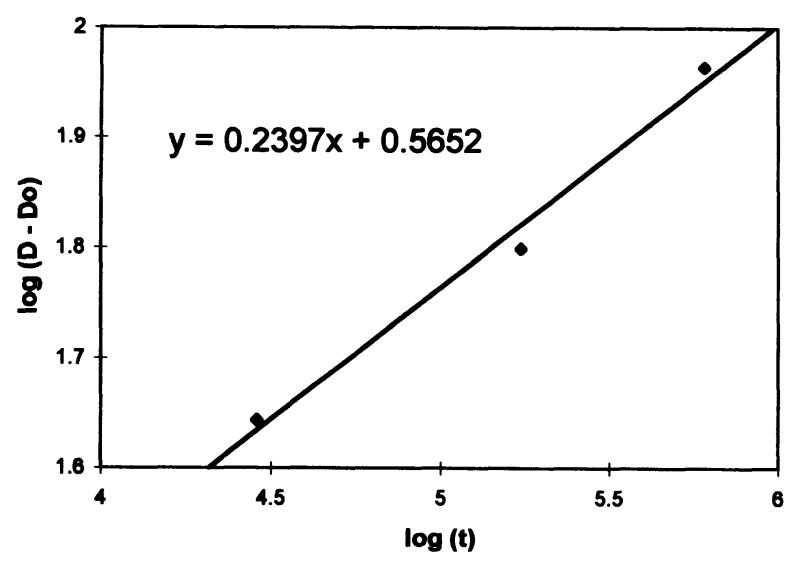

Figure 4 Plot of $\log (D-D o)$ versus $\log (t)$, used to evaluate the grain growth exponent. The equation of the straight line is given on the graph.

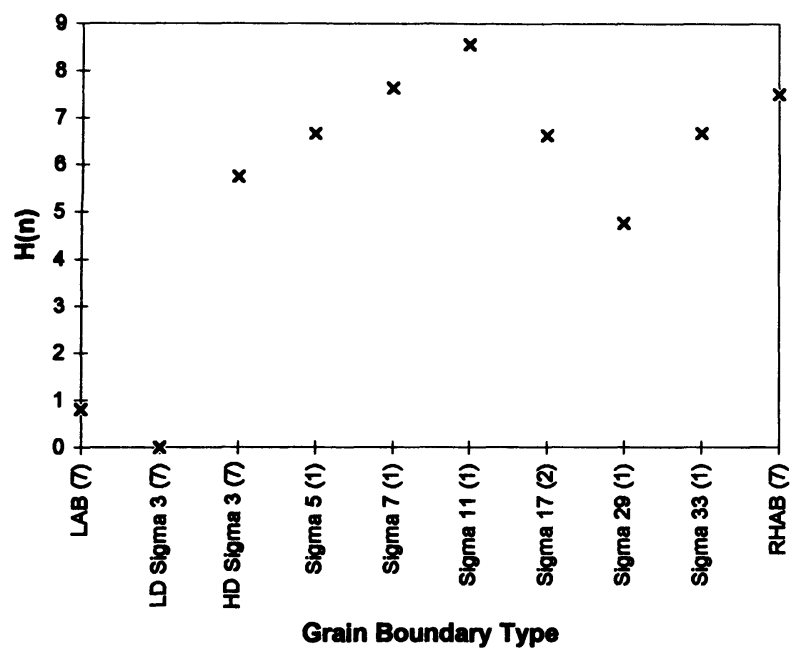

Figure 5 Grain boundary hardening in superpure nickel, as a function of boundary type. The number of boundaries studied is given in parentheses in each case. $H(n)$ is the normalised hardening, given by $(100 \mathrm{dH} / \mathrm{Hg})$, where $\mathrm{Hg}$ is the mean grain hardness and $\mathrm{dH}$ is the difference between the boundary hardness and $\mathrm{Hg}$.

\section{DISCUSSION}

Texture is known to be only one of a number of factors that affect grain boundary misorientations. In addition, it is highly probable that the degree to which texture changes can be applied to the modification of grain boundary structures varies from one material to the next. In the present study we are looking to correlate changes in texture with increased proportions of special boundaries, namely $\Sigma 3 \mathrm{~s}$, or more specifically LD $\Sigma 3$ s. Although a few textures do develop with increased annealing 
time, only the (103)[010] texture changes significantly from sample 3 to $4(2.5 \%$ to 7.2\%) in tandem with the strong increase in LD $\Sigma 3$ proportions (see Figure 3). However, because the increase in (103)[010] texture is not a prodigious one, it was considered wise to conduct a spatial texture analysis about the LD $\Sigma 3$ boundaries in sample 4 , to determine whether or not there is any preference for the (103)[010], or any other, texture in the grains adjoining LD $\Sigma 3$ boundaries. Of the 257 pairs of texture types identified in this procedure, less than 6\% involved a (103)[010] textured grain, which is a lower proportion than in the overall population (7\%). No other strong correlations were found between texture types across LD $\Sigma 3$ boundaries. It must therefore be concluded that under conditions such as those proposed in this work, the grain boundary population in superpure nickel cannot be predicted by simply observing changes in the texture.

Whereas various models have been formulated to explain twin, and especially coherent twin, formation in fcc metals (e.g. Fullman and Fisher, 1951; Gindraux and Form, 1973; Meyers and Murr, 1978), they have all concentrated on how new twin boundaries may be produced from, or introduced into, a locally twin-deficient grain boundary array. (As stated previously, coherent twins are associated with very low deviation from the exact $\Sigma 3$ misorientation (Lim and Raj, 1984b; Randle and Brown, 1989)). The results observed in this work indicate that in addition to twin formation another mechanism can operate. This was proposed in an earlier paper (Thomson and Randle, 1996b) and it involves the 'fine tuning' of HD $\Sigma 3$ s into LD $\Sigma 3$ s, with accompanying reduction in the grain boundary free energy. When studying the effects of strain annealing on commercially pure nickel, the present authors hypothesised that the introduction, and subsequent precipitation, of oxygen at the grain boundaries increased the activation energy for grain/grain boundary rotations above that for annealing twin formation (Thomson and Randle, 1996a). At annealing temperatures of $750^{\circ} \mathrm{C}$ and $850^{\circ} \mathrm{C}$ the latter mechanism was found to dominate. However, when the concentration of second phase particles at the grain boundaries is so low, as is the case for superpure nickel, the precipitation of supersaturated oxygen occurs in a more homogeneous manner. As a consequence, the activation energy for grain/grain boundary rotation is not increased to such a high level, and hence this remains the dominant energy minimization mechanism, rather than annealing twin formation. If annealing twin formation had taken over as the dominant process, a far higher proportion of LD $\Sigma 3$ s would be evident for samples 2 and 3 (i.e. newly generated twins), as the times involved in these two cases are just below and just above those used in the earlier work with the commercial grade material. The conclusion to be drawn from this evidence is that the large numbers of LD $\Sigma 3$ s observed in Figure 3 for sample 4 are produced by fine tuning the existing HD $\Sigma 3$ boundaries, i.e HD $\Sigma 3$ s, with higher associated energy, are evolving to lower energy LD $\Sigma 3$ s.

The energy minimization process involved could occur by making use of any EGBDs remaining after the production of the grain boundary distribution observed for sample 3, which effectively constitutes a metastable grain boundary array in terms of the CSL distribution. Such EGBDs, which are formed via the dissociation of straininduced dislocations, can be absorbed to permit the grain boundaries to rotate slightly. The motivation is still to produce lower energy boundary configurations, and this fine tuning stage achieves this by enabling exact misorientations to be approached. As such an effect is only observed for $\Sigma 3 \mathrm{~s}$, it would appear that other CSL types do not represent significantly lower energy grain boundaries than RHABs. Such a statement is reinforced by the results of the grain boundary hardening study. 
Figure 5 shows that a division can be made, grouping LABs and LD $\Sigma 3$ s into one category (special boundaries) and all other boundaries into another (non-special boundaries). Note that the two defined types of $\Sigma 3$ boundary, with low and high deviation from exact misorientation, have been identified as presenting markedly different hardnesses. The nature of the boundary hardening experiment was such that fairly limited statistics are obtained for each individual boundary. However, any unreliability that this introduces to the testing procedure is reduced by repeating over a number of boundaries of the same misorientation type, suggesting that the results constitute a real effect.

\section{CONCLUSIONS}

1. Changes in texture as a result of strain annealing cannot be used to predict changes in the grain boundary distribution, at least for superpure nickel under these processing conditions.

2. Different grain boundary energy minimization mechanisms are found to be active in superpure and commercially pure nickel. Annealing twin formation can supersede grain/grain boundary rotations more readily in the commercial grade material.

3. Strain annealing can induce significantly increased proportions of special boundaries, even in the absence of extended annealing twin formation. The fine tuning of HD $\Sigma 3 \mathrm{~s}$, with high deviation from exact misorientation, to LD $\Sigma 3 \mathrm{~s}$, with low deviation from exact misorientation, is identified as the active mechanism responsible for this change.

4. Differences in the hardness of various grain boundary types have been identified, with LABs and LD $\Sigma 3$ s hardening to a lesser extent than other boundaries. A marked difference in boundary hardness was found between HD and LD $\Sigma 3 \mathrm{~s}$.

\section{Acknowledgements}

CBT is grateful to the EPSRC for financial support.

\section{References}

Brandon, D. G. (1966) The structure of high-angle grain boundaries. Acta Metallurgica, 14, $1479-1484$.

Chiba, A., Hanada, S., Watanabe, S., Abe, T. and Obana, T. (1994). Relation between ductility and grain boundary character distributions in $\mathrm{Ni}_{3} \mathrm{Al}$, Acta Metallurgica et Materialia, 42, 1733-1738.

Chou, Y. T., Cai, B. C., Romig, Jr., A. D. and Lin, L. S. (1983). Correlation between grain-boundary hardening and grain-boundary energy in niobium bicrystals. Philosophical Magazine A, 47, 363-368.

Crawford, D. C. and Was, G. S. (1991). Grain boundary character distributions in Ni-16Cr-9Fe using selected area channelling patterns: methodology and results. Journal of Electron Microscopy Technique, 19, 345-360.

Fullman, R. L. and Fisher, J. C. (1951). Formation of annealing twins during grain growth. Journal of Applied Physics, 22, 1350-1355.

Gindraux, G. and Form, W. (1973). New concepts of annealing twin formation in face-centred cubic metals. Journal of the Institute of Metals, 101, 85-93.

Lim, L. C. and Raj, R. (1984a). On the distribution of $\Sigma$ for grain boundaries in polycrystalline nickel prepared by the strain-annealing technique, Acta Metallurgica, 32, 1177-1181. 
Lim, L. C. and Raj, R. (1984b). Effect of boundary structure on slip-induced cavitation in polycrystalline nickel. Acta Metallurgica, 32, 1183-1190.

Meyers, M. A. and Murr, L. E. (1978). A model for the formation of annealing twins in f.c.c. metals and alloys. Acta Metallurgica, 26, 951-962.

Murr, L. E., Smith, P. J. and Gilmore, C. M. (1968). Relative interfacial free energies in pure nickel, dispersion hardened nickel, and a precipitation hardened nickel-base alloy. Philosophical Magazine A, 17, 89-106.

Palumbo, G and Aust, K. T. (1990). Grain boundary character distributions in nickel. In Recrystallization '90, edited by T. Chandra, pp. 101-111. The Minerals, Metals and Materials Society.

Pumphrey, P. H. (1976). Special high angle grain boundaries. In Grain Boundary Structure and Properties, edited by G. A. Chadwick and D. A. Smith, pp 139-200. London: Academic Press.

Randle, V. and Brown, A. (1989). Development of grain misorientation texture, in terms of coincident site lattice structures, as a function of thermomechanical treatments. Philosophical Magazine A, 59, 1075-1089.

Thomson, C. B. and Randle, V. (1996a). The effects of strain annealing on grain boundaries and secure triple junctions in nickel 200. In press.

Thomson, C. B. and Randle, V. (1996b). A study of twinning in nickel. In press.

Watanabe, T., Kitamura, S. and Karashima, S. (1980). Grain boundary hardening and segregation in alpha iron-tin alloy. Acta Metallurgica, 28, 455-463. 